\title{
Aa. Vv., Francophonie and its Futures
}

\section{Carminella Biondi}

\section{(2) OpenEdition}

\section{Journals}

\section{Edizione digitale}

URL: https://journals.openedition.org/studifrancesi/4829

DOI: $10.4000 /$ studifrancesi.4829

ISSN: 2421-5856

\section{Editore}

Rosenberg \& Sellier

\section{Edizione cartacea}

Data di pubblicazione: 1 avril 2012

Paginazione: 190-191

ISSN: 0039-2944

\section{Notizia bibliografica digitale}

Carminella Biondi, «Aa. VV., Francophonie and its Futures», Studi Francesi [Online], 166 (I | LVI) | 2012, online dal 30 novembre 2015, consultato il 19 novembre 2021. URL: http://journals.openedition.org/ studifrancesi/4829 ; DOI: https://doi.org/10.4000/studifrancesi.4829

\section{Questo documento è stato generato automaticamente il 19 novembre 2021.}

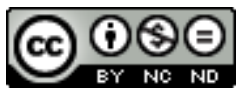

Studi Francesi è distribuita con Licenza Creative Commons Attribuzione - Non commerciale - Non opere derivate 4.0 Internazionale. 


\title{
Aa. Vv., Francophonie and its Futures
}

\author{
Carminella Biondi
}

\section{NOTIZIA}

Francophonie and its Futures, «Australian Journal of French Studies», 1, gennaio-aprile 2011, pp. 118.

1 Presentando nel numero precedente di «Studi Francesi» il volume di Dominique Combe, Les littératures francophones. Questions, débats, polémiques, (2010), avevo cercato di fare il punto del serrato dibattito attorno al concetto di «francofonia», dibattito che è ora rilanciato e approfondito in questo bel numero della rivista australiana. Il dossier si apre con il documentatissimo saggio di Jacqueline DUTTON, Francophonie and its Futures: Utopian, Digital, Plurivocal (pp. 3-18) che costituisce la piattaforma su cui si articola il discorso dei saggi che seguono, perché non solo fa il punto della situazione, anche sullo sfibrante dibattito nominalistico, ma, come ben indica il titolo, prefigura possibili scenari futuri. Segue il saggio di Peter BROWN, "La Francophonie": from the Postcolonial to the Post-Cold War Eras (pp. 19-33), che intreccia il discorso postcoloniale con quello sulla fine della Guerra fredda, giungendo alla seguente conclusione: «It remains to be seen whether institutional Francophonie, such as it evolved as a geopolitical organisation in the post-Cold War period, is able to fulfil the grand aspirations announced for it by Dominique Wolton» (p. 33, l'autore fa qui riferimento al saggio di Wolton, Demain la Francophonie, 2006, che presenta una visione idealistica della Francofonia). Nel terzo articolo, Ibrahima DIALLo fa il punto su Les défis linguistiques et géopolitiques du français en Afrique au Sud du Sahara (pp. 34-46). Diallo prende atto dell'ampliamento dell'area della Francofonia come istituzione (per la quale ormai tutti gli studiosi usano la F maiuscola), che è passata dalla ventina di stati membri del 1975 ai cinquantasei del 2010, ma insiste anche sulla progressiva perdita di presa reale del francese nei paesi africani: «Cependant, ces succès ne doivent pas constituer l'arbre qui cache la forêt: le français recule en terme de prestige, importance et intérêt dans ses bastions africains...» (p. 45). Questa caduta è dovuta per un verso alla nota, folgorante avanzata dell'inglese, ma anche ad una perdita di credibilità della Francia, a causa dei rapporti troppo stretti con 
certi dittatori e delle sue ingerenze negli affari dei paesi africani. Segue un testo che apre un piccolo focus sulla francofonia orientale, in particolare cinese: Perspective d'une francophonie en Orient: un plaidoyer (pp. 47-59). L'autrice, Mathilde KANG, che si limita a presentare solo pochi testi, propone di partire da una recensione di tutte le opere di autori cinesi scritte in francese, al fine di tracciare un panorama esaustivo di questa letteratura non priva di interesse intrinseco e fondamentale per scrivere o riscrivere la storia dei rapporti franco-cinesi. Dall'Oriente si torna in Europa con il saggio di Paul DIRKX, Antinomy and Forms of Literary Migration: the Case of the Belgian Francophone Diaspora (pp. 60-73), che si propone di contribuire «to the study of literary migration within Francophonie, through the example of Belgian-French literary relations», partendo dalla constatazione che la produzione letteraria del Belgio è sempre stata caratterizzata da un alto livello di migrazione verso la Francia, ma che le complesse traiettorie della migrazione restano ancora da studiare. Con il saggio che segue, Destination "littératuremonde"? Travel and Language in "Aux États-Unis d'Afrique" (pp.74-87), si arriva inevitabilmente, come capita ormai in tutti i recenti dibattiti sulla «francofonia», al discusso e fortunatissimo manifesto Pour une littérature-monde en français, apparso su «Le Monde» nel marzo 2007, a cura di Michel Le Bris e Jean Rouaud. L'autore del saggio, Aedín Ní LoINGSIGH, applica qui, retrospettivamente, il concetto di letteratura-mondo al romanzo di Abdouraham A. Waberi, Aux États-Unis d'Afrique, pubblicato nel 2006, che prefigura scenari futuri lusinghieri per l'Africa e molto meno per l'Europa e per l'America. Il critico trova una legittimazione dell'operazione compiuta, se ce ne fosse bisogno, nel fatto che Waberi ha firmato il manifesto e contribuito all'antologia, pubblicata alcuni mesi dopo da Gallimard con il titolo Pour une littérature-monde. Allargando, in conclusione, il discorso agli scrittori africani, il critico afferma: "Whether or not they accept that their future is part of a littérature-monde, the challenge for African writers is to remain aware of this privilege, to continue to relativise its significance in relation to other forms of mobility, and to determine whether the concerns, hopes and fears of the global world correspond to the local worlds they leave behind» (pp. 86-87). Dopo un saggio di Charles FORSDICK, Mobilising French Studies (pp. 88-103) che analizza il manifesto di Stephen Greenblatt, "mobility studies manifesto", del 2010, confrontandolo con il più noto e già citato Pour une littérature-monde, il volume si conclude con un studio quasi obbligato, di Dominic THOMAS, sul passaggio dalla letteratura-mondo all'identità-mondo: From "WorldLiteratures" to "World-Identities" (pp. 105-116), seguendo il percorso fatto dai curatori del primo manifesto, Michel Le Bris e Jean Rouaud, che hanno coordinato, nel 2010, sempre per Gallimard, un volume collettivo, con molti degli autori che avevano partecipato all'operazione precedente, dal titolo rimbaldiano Je est un autre. Pour une identité-monde. Un bel numero che, pur nella varietà dei contributi, riesce a ricostruire un percorso e apre, come si propone il titolo, spiragli sui «futuri» della francofonia. 